Center for

Mathematical Economics

Working Papers

June 2016

\title{
A Note on the Perpetual American Straddle
}

Lazar Obradović

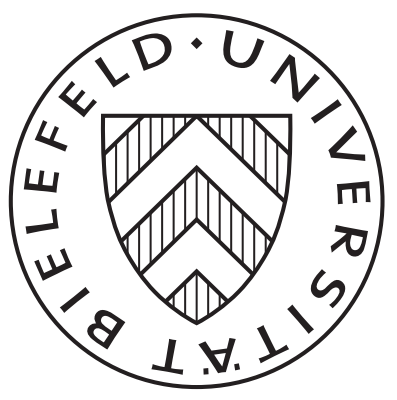

Center for Mathematical Economics (IMW)

D-33615 Bielefeld · Germany

e-mail: imw@uni-bielefeld.de 


\title{
A Note on the Perpetual American Straddle
}

\author{
Lazar Obradović*
}

June 10, 2016

\begin{abstract}
The value and the optimal exercise time of the perpetual American straddle is characterized by the unique solution of a single non-linear equation with one unknown variable.
\end{abstract}

\section{Introduction}

American options form an important class of derivatives; their perpetual versions can frequently be priced explicitly in the standard Black-Scholes model [1]. This is the case with the American straddle - a portfolio consisting of a put and call option on the same underlying with the same strike price. Pricing of the American straddle has been studied using different approaches and tools: in [4] by applying the theory of Laplace transforms, in [5] by transforming the problem to a "generalized parking problem", in [6] by exploiting "an analogy with asymmetric rebates of double knock-out barrier options", in [7] "by means of the Esscher transform and the optional sampling theorem", and, more recently, by using a combination of several optimization techniques [2], [3]. In all of these papers the value function and the optimal excercise time ${ }^{1}$ of the perpetual American straddle are characterized by the solution of a non-linear system of equations consisting of (at least) two equations.

In this note, we show that the value function and the optimal excercise time of the American straddle can be characterized via a unique solution of a single one-variable equation; the solution lies in the interval $(0,1)$. We do so by using one of the classical ${ }^{2}$ optimal stopping theory approaches: HJB equation and smooth-fit principle in combination with a verification theorem. This leads to a system of non-linear equations that can, by appropriate transformations, be reduced to a single equation. To the best of our knowledge this is the first time that such a one-equation characterization of the value and the optimal excercise time of the perpetual American straddle is obtained.

\footnotetext{
*Center for Mathematical Economics (IMW), Bielefeld University, Germany, and Faculty of Natural Sciences and Mathematics, University of Montenegro, Montenegro. Email: lazaro@ac.me

${ }^{1}$ It is obviously optimal to excercise only one of the options that straddle consists of; this is the reason why we speak of exercising the straddle.

${ }^{2}$ See [11], [8], [9].
} 


\section{Result}

Let the price process $S_{t}$ be a a geometric Brownian motion, $d S_{t}=\alpha S_{t} d t+$ $\sigma S_{t} d B_{t}$, where $\alpha \in \mathbb{R}$ and $\sigma \in \mathbb{R}$ are known constants. The American straddle yields a payoff $f\left(t, S_{t}\right)=e^{-r t}\left|S_{t}-I\right|$ when exercised at time $t$, where $I>0$ is the strike price and $r \leq \alpha$ is a given ${ }^{3}$ discount rate.

The value of the perpetual American straddle at time $t$ is given by

$$
V_{t}=\underset{\tau \in \mathcal{T}_{t}}{\operatorname{ess} \sup } E\left[e^{-r \tau}\left|S_{\tau}-I\right|\right]
$$

where $\mathcal{T}_{t}$ is a set of all stopping times $\tau \geq t$. Our goal is to find the explicit representation of the value function $v(t, x)$ such that $v\left(t, S_{t}\right)=V_{t}$ and an optimal stopping time $\tau^{*}$ such that $V_{\tau^{*}}=E\left[e^{-r \tau^{*}}\left|S_{\tau^{*}}-I\right|\right]$ holds.

Becuse of the form of the payoff function $f$ we expect that there will be two values, $x_{1}$ and $x_{2}, x_{1}<I<x_{2}$, such that it is optimal to exercise the put (call) option when the value of $S_{t}$ goes beneath $x_{1}$ (above $x_{2}$ ). This means that the optimal stopping time will be the first exit time from inteval $\left[x_{1}, x_{2}\right]$. Since the function $f=\left(e^{-r t}\right)|x-I|$ is continuous, we expect the value function $v$ to be smooth. We also expect that it will satisfy the Hamilton-Jacobi-Bellman (HJB) equation:

$$
\max _{(t, x) \in[0,+\infty] \times \mathbb{R}}\left\{f(t, x)-v(t, x), v_{t}(t, x)+\mathcal{L} v(t, x)\right\}=0,
$$

where $\mathcal{L}:=\alpha \frac{\partial}{\partial x}+\frac{\sigma^{2}}{2} \frac{\partial^{2}}{\partial x^{2}}$ is a differential operator.

We "guess" that the the value function is of the form $v(t, x)=e^{-r t} \varphi(x)$; this is a well known approach when dealing with time-discounted optimal stopping problems. Because of the HJB equation we expect that on the continuation region the function satisfies $v_{t}(t, x)+\mathcal{L} v(t, x)=0$, which, after canceling $e^{-r t}$, gives:

$$
r \varphi-\alpha x \varphi^{\prime}(x)-\frac{1}{2} \sigma^{2} \varphi^{\prime \prime}(x)=0 .
$$

The last equation is a well known Cauchy-Euler ODE and it's solution is:

$$
\varphi(x)=A x^{\lambda}+B x^{\mu}
$$

where $A$ and $B$ are two unkown constants and $\lambda$ and $\mu$ solve the characteristic equation $r-\alpha m-\frac{1}{2} \sigma^{2} m(m-1)=0$. It can be easily $\operatorname{seen}^{4}$ that $\lambda>1$ and $\mu<0$. We conclude that function $v$ should be of the form:

$$
v(t, x)= \begin{cases}e^{-r t}(I-x), & 0<x \leq x_{1} \\ e^{-r t}\left(A x^{\lambda}+B x^{\mu}\right), & x_{1} \leq x \leq x_{2} \\ e^{-r t}(x-I), & x>x_{2}\end{cases}
$$

\footnotetext{
${ }^{3}$ Inequality $r \leq \alpha$ is a standard assumption; see for example [12].

${ }^{4}$ since: $\lambda=\frac{1}{2}-\frac{\alpha}{\sigma^{2}}+\sqrt{\left(\frac{1}{2}-\frac{\alpha}{\sigma^{2}}\right)^{2}+\frac{2 r}{\sigma^{2}}}$ and $\mu=\frac{1}{2}-\frac{\alpha}{\sigma^{2}}-\sqrt{\left(\frac{1}{2}-\frac{\alpha}{\sigma^{2}}\right)^{2}+\frac{2 r}{\sigma^{2}}}$
} 
where $A, B, x_{1}, x_{2}$ are unknown constants. Since we expect the value function to be smooth we will try finding these constants by requiring continuity and differentiability in points $x_{1}$ and $x_{2}$ (smooth pasting conditions). It is already clear that, should we find such constants, the above function $v(t, x)$ will indeed be a value function. This can be seen by checking the function $v$ via one of the well known verification theorems for the optimal stopping of diffusions ${ }^{5}$.

Smooth pasting condtions, after cancelling out $e^{-r t}$, yield the following system:

$$
\begin{aligned}
I-x_{1} & =A x_{1}^{\lambda}+B x_{1}^{\mu} \\
-x_{1} & =A \lambda x_{1}^{\lambda}+B \mu x_{1}^{\mu} \\
x_{2}-I & =A x_{2}^{\lambda}+B x_{2}^{\mu} \\
x_{2} & =A \lambda x_{2}^{\lambda}+B \mu x_{2}^{\mu}
\end{aligned}
$$

The above system is higly nonlinear. After several transformations and introduction of a new variable the above system can be reduced to a one-dimensional system which has a unique solution in the interval $(0,1)$. It turns out that this solution completely characterizes the above problem. This is our main result and we formulate it precisely in the following proposition:

Proposition 1. The value process of the perpetual American Straddle $V_{t}$ defined in (1) satisfies the equality $V_{t}=v\left(t, S_{t}\right)$ for the function $v$ as defined in (5) where

$$
\begin{array}{rlrl}
A=\frac{1}{\mu-\lambda}\left((1-\mu) x_{1}^{1-\lambda}+\mu x_{1}^{-\lambda}\right) ; & B & =\frac{1}{\lambda-\mu}\left((1-\lambda) x_{1}^{1-\mu}+\lambda x_{1}^{-\mu}\right) \\
x_{1}=\gamma x_{2} ; & x_{2}=\frac{\mu I}{\mu-1} \frac{1+\gamma^{-\lambda}}{1+\gamma^{1-\lambda}}
\end{array}
$$

and $\gamma$ is the unique number in $(0,1)$ satisfying

$$
\frac{\mu I}{\mu-1} \frac{1+\gamma^{-\lambda}}{1+\gamma^{1-\lambda}}-\frac{\lambda I}{\lambda-1} \frac{1+\gamma^{-\mu}}{1+\gamma^{1-\mu}}=0 .
$$

The optimal stopping time is the first exit time from the region $\left[x_{1}, x_{2}\right]$ :

$$
\tau^{*}=\inf \left\{t \geq 0 \mid S_{t} \notin\left[x_{1}, x_{2}\right]\right\} .
$$

Proof. In order to prove the porposition it is, by construction of the value function $v$, sufficient to prove that unique solution of the system $(6)-(9)$ is the one given in the formulation of the proposition. The proof consists of reducing the

\footnotetext{
${ }^{5}$ The simplest form of such a theorem available to author appears (in a more general version) in [10] and is derived from [11]. Another formulation is available in ch.4 of [8]. Due to the fact that this function coincides, piecewise, with discounted 'quasi polinomials', conditions of each of the standard verification theorems are easily satisfied.
} 
system to equation (10), and proving that the solution of the latter is unique on the interval $(0,1)$.

First we comment on the uniqueness of the solution of the system of equations (6)-(9). Due to the uniqueness of the value function of the optimal stopping problems the solution of the system above must be unique. Indeed, two different solutions of the system $(6)-(9)$ would lead to two functions $v_{1}$ and $v_{2}$ both of which would satisfy the verification theorem and the equation $v_{1}\left(t, S_{t}\right)=v_{2}\left(t, S_{t}\right)$ would holds almost surely, which is clearly impossible.

We now turn to proving the existence. We eliminate variables $A$ and $B$ by treating equations $(6)-(7)$ as a two dimensional linear system. Determinant of that system is $D=x_{1}^{\lambda+\mu}(\mu-\lambda)$ which is always different from zero ${ }^{6}$, so variables $A$ and $B$ are uniquely determined by:

$$
A=Q\left(x_{1} ; \mu, \lambda\right) ; \quad B=Q\left(x_{1} ; \lambda, \mu\right)
$$

where $Q(x ; \mu, \lambda):=\frac{1}{\mu-\lambda}(1-\mu) x^{1-\lambda}+\mu x^{-\lambda}$ is a function introduced for notational purposes. Similarly, equations $(8-9)$ allow us to obtain expression for variables $A$ and $B$ in terms of $x_{2}$ :

$$
A=-Q\left(x_{2} ; \mu, \lambda\right) ; \quad B=-Q\left(x_{2} ; \lambda, \mu\right)
$$

Equating the expressions for $A$ and $B$ we obtain the following nonlinear system with two equations and two variables, $x_{1}$ and $x_{2}$ :

$$
Q\left(x_{1} ; \mu, \lambda\right)+Q\left(x_{2} ; \mu, \lambda\right)=0 \quad Q\left(x_{1} ; \lambda, \mu\right)+Q\left(x_{2} ; \lambda, \mu\right)=0
$$

Due to the nice form of the above system, we immediately see that if $\left(x_{1}, x_{2}\right)$ is it's solution so is $\left(x_{2}, x_{1}\right)$. This means that there is a unique solution pair satisfying $x_{1}<x_{2}$, and it will be the unique solution that we are looking for. Because of this we introduce a variable $\gamma$ such that $x_{1}=x_{2} \gamma$. Since inequality $0<x_{1}<x_{2}$ holds, we have $\gamma \in(0,1)$. The right hand side of the first equation of the system (13) can now, after some simple calculations, be writen as:

$$
Q\left(x_{2} \gamma ; \mu, \lambda\right)+Q\left(x_{2} ; \mu, \lambda\right)=(1-\mu) x_{2}^{1-\lambda}\left(1+\gamma^{1-\lambda}\right)+\mu x_{2}^{-\lambda}\left(1+\gamma^{-\lambda}\right) .
$$

from which we obtain:

$$
x_{2}=\frac{\mu I}{\mu-1} \frac{1+\gamma^{-\lambda}}{1+\gamma^{1-\lambda}}
$$

Similarly, by changing $x_{1}=x_{2} \gamma$ in $Q\left(x_{1} ; \lambda, \mu\right)+Q\left(x_{2} ; \lambda, \mu\right)=0$ after multiplication with $x_{1}^{-\mu}$ we obtain:

$$
x_{2}=\frac{\lambda I}{\lambda-1} \frac{1+\gamma^{-\mu}}{1+\gamma^{1-\mu}} .
$$

\footnotetext{
${ }^{6}$ Because $x_{1}>0$ and $\lambda>\mu$.
} 
Equating the two obtained expressions for $x_{2}$, after rearanging and cancelling out parameter $I$, we obtain the one-dimensional equation (10), stated in the formulation of the proposition.

It remains to prove that there exists a unique solution of equation (10) in the interval $(0,1)$. Indeed, if we denote the left hand side of the equation with $h(\gamma)$ it is obvious that function $h$ is continuous on $(0,1)$, and it is easy ${ }^{7}$ to check that $h(1)<0$, and $\lim _{\gamma \rightarrow 0+} h(\gamma)=+\infty$. We can thus conclude that a solution exists on the interval $(0,1)$, and it's uniqueness is consequence of the argument from the beginning of the proof.

\section{References}

[1] Black, Fischer; Myron Scholes (1973). "The Pricing of Options and Corporate Liabilities" Journal of Political Economy 81 (3): 637654.

[2] Jukka Lempa, A note on optimal stopping of diffusions with a two-sided optimal rule. Operations Research Letters, Volume 38, Issue 1, January 2010, Pages 11-16,

[3] Damien Lamberton and Mihail Zervos, On the optimal stopping of a onedimensional diffusion, Electronic Journal of Probability, Volume 18, 2013

[4] G. Alobaidi and R. Mallier, Laplace transforms and the American straddle. Journal of Applied Mathematics Volume 2 (2002), Issue 3, Pages 121-129

[5] Beibel, M. and Lerche, H.R., A new look at optimal stopping problems related to mathematical finance. Statistica Sinica Volume 7 (1997), Pages 93-108

[6] Franck Moraux, On Perpetual American Strangles. The Journal of Derivatives Summer 2009, Vol. 16, No. 4: pp. 82-97

[7] Gerber, H.U. and Shiu, E.S.W., Martingale approach to pricing perpetual American options. Astin Bulletin Vol. 24 (1994), No. 2: pp. 195-220

[8] Oksendal, B., Stochastic Differential equations, 5th ed. Vorlag-Springer 2003.

[9] Pham, H., Continuous-time Stochastic Control and Optimization with Financial Applications Springer 2009.

[10] Xue Cheng and Frank Riedel, Optimal Stopping under Ambiguity in Continuous Time. Mathematics and Financial Economics 7, No. 1, 2013, 29-68

[11] N. V. Krylov, Controlled Diffusion Processes Spriger Verlag 2008

[12] Shiryaev, Albert N. Essentials of stochastic finance: facts, models, theory. Vol. 3. World Scientific Publishing Company Incorporated, 1999.

\footnotetext{
${ }^{7}$ Because $\lambda>1$ and $\mu<0$.
} 